\title{
Dynamic and Scalable Provisioning in Wireless Mesh Networks to Efficiently Support Multi-User Killer- Applications with High-Demand of Resources
}

\author{
Augusto Neto ${ }^{1}$, Eric Patrick ${ }^{2}$, Eduardo Cerqueira ${ }^{3}$, Danielo Gomes ${ }^{1}$, Rui Aguiar ${ }^{4}$ \\ ${ }^{1}$ Federal University of Ceará (UFC), Brazil \\ ${ }^{2}$ Federal University of Goiás (UFG), Brazil \\ ${ }^{3}$ Federal University of Pará (UFPA), Brazil \\ ${ }^{4}$ Institute for Telecommunications (IT), Universidade de Aveiro (UA), Portugal \\ augusto.deti@ufc.br, ericpatrick15@gmail.com,cerqueira@ufpa.br, ruilaa@ua.pt
}

\begin{abstract}
The success of mobile and ubiquitous computing, coupled with the increasing demand for applications with high Quality of Service (QoS) and Quality of Experience (QoE) requirements, has brought great challenges to the future access networks. Thus, wireless mesh networks distinguish due to its flexibility, redundancy, low-cost and broadband capacity. However, aspects as scalability, availability and reliability, are still challenging. Following the limitations of existing solutions, this paper proposes the Multi-Service Resource Allocation in Wireless Mesh Networks (MIRA-WMN) for provisioning resources of wireless mesh networks compliant with IEEE 802.11e/s standard. The MIRA-WMN proposes a single solution to integrate QoS and connectivity resource control to efficiently support multi-user sessions. The MIRA-WMN was evaluated by simulations, which demonstrated its benefits in the data and control plane, as well as user's perception.
\end{abstract}

\section{Keywords: Wireless mesh, QoE, QoS, Resource allocation}

\section{INTRODUCTION}

The increasing need of ubiquitous access (at any place and time) to the Internet, combined to the increasing demand of time-sensitive contents (e.g., real time multimedia) and wireless devices, is turning mobile computing into an everyday tool in people's lives. The abundance of emerging multi-user high resource demanding session (a.k.a. killer-applications) has lured more and more users, thus requiring the application of Quality of Service (QoS) techniques to ensure dependable endto-end data transmission at reliable Quality of Experience (QoE) levels. Besides basic operations (e.g., traffic differentiation/shaping, congestion control), QoS application involves at least admission control, resource provisioning and resilience without interfering with the system scalability, which can occur by system overhead (signaling, CPU, memory).

Among the current wireless access technologies available, the IEEE 802.11 has become dominant. The IEEE 802.11s standard [1] developed an emerging pattern for multihops communication at MAC layer (relaying) to increase wireless area capabilities. The HWMP (Hybrid Wireless Mesh Protocol) provides a route selection scheme enabling Mesh Points (MPs) relaying packets over the mesh network. The IEEE 802.11e [2] defines a set of features embedding Access Points with QoS support (QMP). A new coordination function (Hybrid Coordination Function - HCF) was defined for using only in a basic set of services supporting two types of operation, EDCA (Enhanced Distributed Channel Access) and HCCA (HCF Controlled Channel Access). The EDCA is based on contention and works simultaneously with the HCCA. Whereas the EDCA was designed to enable traffic (Traffic Streams - TS) with differentiation of users priorities by allocation of opportunistic transmission (TXOP), the HCCA traffic is parameterized. The Access Category (AC), the class of service concept of IEEE 802.11e, determines the behavior of TS within a QMP.

The TS setup is requested to the HCF on a QMP by an ADDTS message carrying Traffic Specifications (TSPEC). The TSPEC provides QoS parameters to the HCF (average bit-rate, nominal SDU size, minimal PHY rate, delay/loss tolerance and maximum service interval) and the selected AC, so this is able to configure the packet scheduler for bandwidth reservation. The HCF may admit or reject a new TS request by the verification of admission control according with the requested TSPEC and current network conditions. For the multi-user support, QMPs encapsulate referred packets into multicast frames for member propagation over appropriate channels. The multicast processing is not sensitive to QoS, which means setting up multicast channels without considering TSPECs.

We claim that the integration of IEEE 802.11 "e" and "s" defined tools is of paramount to the efficient support of multiuser killer-application sessions over WMNs. However, IEEE $802.11 \mathrm{e} / \mathrm{s}$ are not autonomous, requiring cooperation with external mechanisms to provision enforce resources as demanded [3] while keeping the system performance and QoE. To that, this paper proposes the MIRA-WMN (Multi-service Resource Allocation in Wireless Mesh Networks), yet another QoS controller for IEEE 802.11 compliant networks. The MIRA-WMN distinguishes itself by a dynamic approach, spread and scalable to control QoS-aware multi-user wireless mesh connectivity, offering a better planning on the capacity and a systematized access to the network resources. Moreover, MIRA-WMN supports a low-cost resilient strategy, for intermittence of sessions with excellent perception of quality for multi-users and network performance.

The remaining is organized as follow. Section 2 provides a related work-study. Section 3 describes the MIRA-WMN proposal, while Section 4 the simulation results. Finally, Section 5 concludes the study as well points out future works. 


\section{RELATED WORK}

The study on some works related to what is considered relevant concerning provisioning of resources for admission of killer-application sessions, is described as follow. QoS routing protocols, such as MARIA (Interference-Aware Admission Control and QoS Routing in Wireless Mesh Networks) [4] and QASR (QoS Aware Stable path Routing) [5], aim at establishing routes in WMNs and ad-hoc networks, respectively. With MARIA, each node demanding routing performs the admission control of the flow based on the local residual bandwidth and surrounding nodes. However, MARIA is not recommended for applications sensitive to delays as only the bandwidth is taken as routing metric. On the other hand, in networks with QASR, the adaptive routing is based on signal stability at selecting paths that may survive for long time periods. Nevertheless, the QASR is complex and demands an exceeding signaling exchange to manage the system. None of the solutions mentioned above support multicast.

The ASAP (Adaptive Reservation and Preallocation (ASAP) [6] comes as a second proposal. It reserves bandwidth, by signaling among elements in the route of multimedia flows in real time. Likewise MARIA protocol, the ASAP mechanism only considers the bandwidth as a way to ensure QoS, and also produces a large amount of signaling in the network. Moreover, ASAP's soft reserves may waste the bandwidth, once they are reserved but not allocated.

The WIRA (WiMAX Resource Allocation Control) [7] is a proposal for provisioning resources in WiMAX networks. The WIRA provides bandwidth provisioning per class of service, as well as sessions QoS mapping/adaptation. The WIRA provides an efficient QoS approach for WiMAX wireless networks, however it is not suitable for mesh networks as it is only supported in the access point directly connected to user's.

The study on the related works shows that none of the proposals mentioned above meets the requirements for provisioning resources of multi-user killer-application sessions in WMNs, as they do not support QoS-aware IP Multicast over mesh channels, or produce over-signaling.

\section{GENERAL OVERVIEW OF MIRA-WMN}

The Multi-service Resource Allocation in Wireless Mesh Networks (MIRA-WMN) handles network resources to enable multi-user killer-applications over WMN channels, with QoS guaranteed over the time while keeping system performance. To that, MIRA-WMN provisions and enforces network resources, namely to determine amount of resources for further allocation and to configure network elements as required. The network resources are classified into QoS (per-AC bandwidth) and Connectivity (multicast channels). The MIRA-WNM provides a single solution to control network resources in the same way, where its functionalities are implemented on software agents hosted in all QoS-aided Mesh Points (denoted in this paper as QMPs) of the wireless network.

The MIRA-WMN architecture is composed of two elements, namely MIRA-WMN Resource Controller (MIRAWMN-RC) and MIRA-WMN Protocol (MIRA-WMN-P), and state tables. Fig. 1 shows MIRA-WMN architecture interfacing with IEEE 802.11e/s.
The internal and external interfaces enable local interaction among the architecture elements or to expose its functionalities to schemes and patterns outside the architecture, respectively. The MIRA-WMN-RC is accountable for the provisioning and enforcing of QoS and Connectivity resources. For such, the MIRA-WMN-RC uses interfaces with mechanisms supported by IEEE $802.11 \mathrm{e} / \mathrm{s}$ standard and accordingly. The MIRAWMN-P provides the architecture signaling. To reduce the protocol complexity, MIRA-WMN-P provides only two types of signaling message: RESERVE and RESPONSE. The messages are passed on by the HWMP, which each QMP visited inspects its header to define the actions to be taken.

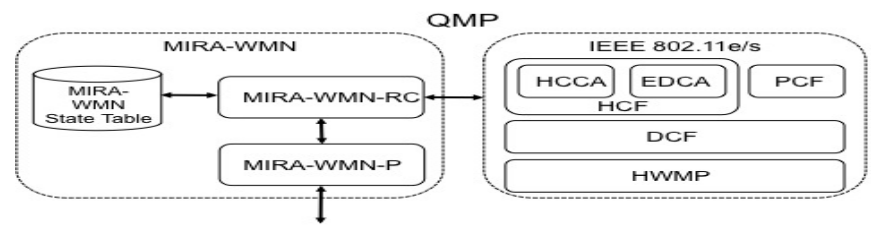

Figure 1. MIRA-WMN Architecture and interface with IEEE 802.11e

The RESERVE message signs to the MIRA-WMN agents in the QMPs toward the QoS-aware stations (QSTAs) to install, modify, release, or collect the QoS and Connectivity current state. Based on the QoS parameters found in the TSPEC, the $\mathrm{HCF}$ is accountable for establishing QoS-guaranteed TCs and assign the frames to their respective queues (classification). Moreover, the MIRA-WMN agents in the ingress QMPs regularly send RESERVE messages that are accountable for requesting refresh of the state previously installed, as well to collect QoS data to update the state tables.

The RESPONSE message is mainly responsible at providing feedback to the previous operation requestor. The MIRA-WNM-RC also uses the RESPONSE message to request release of resources in the upstream downstream direction (clear request for release) or upstream (when the resource allocation failures). As last attempt, the QMP unable to admit a TS immediately creates a RESPONSE message and sends it in the reverse path, which was made by the RESERVE message associated with; thus, all visiting agents release the resources pointed out in the TSPEC object.

To avoid bandwidth monopoly (starvation) of TSs, the MIRA-WMN is based on reserve limits (max and min) manually configured in each AC by the network administrator, which may hold local policies and agreement to the service level (this definition is not the focus of this work). It is not up to the MIRA-WMN to establish the amount of necessary resources for a requesting session, but to perform properly as requested by an external trigger. This work does not focus on defining absolute values for some variables, such as bandwidth. The MIRA-WMN-RC manages the following mechanisms:

- AC Mapping: Responsible for mapping the session's QoS requirements in the most appropriate IEEE 802.11e AC (detailed in [9]). This scheme chooses the most suitable AC to a requesting session by mapping the QoS parameters provided during the request for reestablishment of the session and the list of ACs available in the wireless link, with its current QoS abilities; 
- Admission Control: Responsible for checking, locally, the bandwidth available in the AC selected by the session, taking in account the amount desired and the value of the reserve limit configured. If successful, the session is admitted, otherwise, it is denied. The admission control is essential to the efficiency of resource enforcement and mitigation of the network degradation as a whole;

- Resource enforcement: Responsible for establishing the QoS and Connectivity resources demanded by a session. According to what was previously mentioned, the QoS enforcement in the wireless link is made by explicit request to the local HCF module for setup (ADDTS messages encapsulating a TSPEC) or release (DELTS messages encapsulating a TSPEC) of bandwidth-guaranteed TSs. The Connectivity scheme is designated for the installation, maintenance, or removal of multicast state along the source-to-destination multicast wireless mesh channel.

In the next we provide a use case envisioning to clarify MIRA-WMN operations within a wireless mesh system. In this sense, consider Fig. 2, which shows the MIRA-WMN functionalities to establish a session on a generic scenario compliant to the IEEE $802.11 \mathrm{e} / \mathrm{s}$ standard.

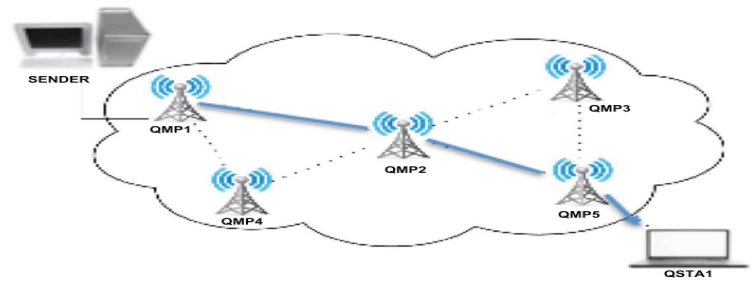

Figure 2. Generic scenario of MIRA-WMN in a IEEE 802.11e/s networks 1) Establishement of Session

To request a session to be established, an application in the sender node activates the MIRA-WMN agent in the neighboring ingress QMP (QMP1, in this case) with SessionSetup.Request message (specified by the external interface) for each flow composing the session (S1). The message must carry: (i) IP address of destination node (QSTA1, in this case); (ii) indication if it is a unicast or multicast session; (iii) session identifier (S1); (iv) flow identifier (F1); (v) and the QoS requirements of the session (bandwidth, max rates of delay, loss and jitter). First, the MIRA-WMN-RC in QMP1 checks in its local state tables if the $\mathrm{S} 1$ is already activated. If yes, the sender request is denied through a primitive SessionSetup.Response, otherwise, the procedure goes on. If it is a multicast session, the MIRA-WMN-RC in QMP1 interacts with a standard mechanism for dynamic allocation of addresses (as Error! Reference source not found.), requesting a local multicast address.

Afterwards, the MIRA-WMN-RC activates the mapping mechanism to generate a TSPEC (with the most suitable AC for S1). After that, the admission control is activated, which checks if the maximum $\mathrm{AC}$ reservation limit was exceeded. If not, the local HCF is activated with an ADDTS.Request with the TSPEC of S1. After successful admission control, the HFC sends an ADDTS.Response to the local MIRA-WMN-RC informing that the resources were successfully enforced (considering that S1 is allocated to a TS with the amount of bandwidth with indicated reserved along the session length). Afterwards, the state table is updated with information on S1: reserved bandwidth, IP of ingress QMP (local IP) and multicast channel allocated. Then, the MIRA-WMN-RC in QMP1 triggers the MIRA-WMN-P to send the RESERVE message properly filled of: IP of destination (QSAT1); IP source (QMP1, unalterable until the end); TSPEC (bandwidth, AC, current capacities of QoS in QMP1, maximum delay and loss); list of QMPs (RSVPATH field, with IP of QMP1).

Each QMP between QMP1 and QSTA1 (QMP2 and QMP5) picks up the RESERVE message (as the IP header flag IP-router alert of the message is on) and performs as QMP1. In the end, the QMP5 composes a RESPONSE message with: destination IP (QMP1); TSPEC changes only if the local QoS capacities are less than the previous QMP's (to ensure information of the bottleneck only); INFOSPEC (111 - OK); and RSVPATH (QMP1; QMP2; QMP5).

After receiving the RESPONSE message, the MIRAWMN-RC in QMP1 updates the S1 information in the state table: the IP address of the QMPs indicated in RSVPATH, found out while the RESERVE message was being sent (found in the RSVPATH field); and the QoS capacities in the bottleneck of the path found (in TSPEC). Finally, the MIRAWMN-RC sends a Session-Setup.Response for the requesting application of S1 (Source) informing that the session was admitted. Observe that the operations are not held end-to-end but border-to-border (i.e., ingress-QMP to egress-QMP), which means that the multicast control is not overcharged according to the number of users (as happens with IP multicast), once the whole operation is ignored if the session is already on.

\section{2) Session Termination}

The MIRA-WMN defines that resources must be immediately available when a session in under termination. The MIRA-WMN decides to end a session if: (i) it is denied by the admission control in a QMP; (ii) when de session ends; (iii) or if there is no user on at a certain egress node. Thus, the immediate release of recourses of an ended session aims at optimizing the blocking probability and enhancing the network performance. However, this operation must be carefully done if the situation (iii) occurs, as the resource releasing on all nodes will disconnect users that are interested in the session yet. To avoid this, the MIRA-WMN defines that the resources can only be released from a branching point for a specific destination.

When an application whishes to request release of resources relating a session, it sends a Session-Delete.Request message to the neighboring ingress QMP including: IP of access QMP, indication of multicast connection, and Session/flow ID. After receiving the request, the MIRA-WMN-RC in the ingress QMP gets information on the session request (TSPEC, etc.) indexing the session ID in the local state table. After that, the MIRAWMN-RC checks if the indicated flow is on. Otherwise, the requesting application is informed, if not, the MIRA-WMN-RC checks if the local QMP is a branching point. Considering that the session indicated is locally on and the local QMP is not a ramification point, the MIRA-WMC-RC ignores the operation and triggers the MIRA-WMN-P to send a RESERVE message (with flag $\mathrm{d}$ on) to find out if the session is on in the remaining nodes of the path and if any of them is a branching point of the 
multicast channel. If the MIRA-WMN-RC realizes that the indicated session is locally on and that the QMP is not a branching point, the operation to release resources is ignored, and this procedure is repeated. Otherwise, the MIRA-WMN$\mathrm{RC}$ releases the resources and updates the input in the state table related to the interface obtained. All the following nodes will do the same until it reaches the egress QMP.

Besides releasing the network resources indicated and upgrading the state table in the network interface obtained, the MIRA-WMN-P sends a RESPONSE message for the ingress QMP. After receiving a successful RESPONSE, the MIRAWMN-P triggers the MIRA-WMN-RC, which will remove the resources related to the indicated flow from the state table.

\section{3) Resilience}

To raise the reliability and availability of resources in WMNs, the MIRA-WMN provides a low-cost resilience support. The concept focuses on the fact that the MIRA-WMN agent monitors the channels status in each active QMP in the network. If the channel break is detected, the immediately affected QMP agent creates and sends a RESPONSE message (with the failure code in the INFOSPEC field) to the ingress QMPs booked in local state tables (known by RESERVE messages previously sent to establish ongoing sessions). When such message is received, each ingress QMP indexes it in the local state tables the paths affected by the QMP with failure, as well as the sessions associated with these paths. After that, the MIRA-WMN creates a RESERVE message to each session affected, and sends it toward the QSTA.

The regular procedure to establish the session is accountable at finding a new path, and then the sessions are properly re-routed by the intra-domain routing approach (HWMP) while the MIRA-WMN provides QoS-enabled multicast support. For state maintenance, MIRA-WMN follows a soft-state approach. When a state is not updated in three periods of refresh signaling ( 30 seconds by default), and having active sessions related to a path with this QMP, the ingress QMP starts the resilience procedure mentioned above, and the difference is in starting it without clear signaling (RESPONSE) from the network, once the node is off.

\section{EVALUATION OF THE MIRA-WMN}

The impact of MIRA-WMN, both for data or control plans for provisioning resources in IEEE $802.11 \mathrm{e} / \mathrm{s}$ networks, was evaluated through simulations using the tool Network Simulator v2 (NS2). To simulate sessions with high requirements and check on the impact of the proposal according to the user's experience, the simulation model was configured with multimedia sessions and background traffic, and the analyses were held through the Evalvid Tool. Once the NS2 IEEE 802.11 agent is incompatible with multicast protocols, the study considered unicast sessions only.

The simulation model was configured with a random topology with QMPs and QSTAs holding transmission capacity of $11 \mathrm{Mbps}$. To simulate multimedia sessions, 65 CBR flows mapped in the AC VI (video), with transmission rate of $256 \mathrm{kbps}$, were used. The continuous flow mapped in class AC_BE was configured for background traffic to exceed in $50 \%$ the network capacity. The AC_BE has a $40 \%$ of local link capacity as reservation limit, while each AC_VI has $20 \%$.
The lifetime of the flows were randomly distributed, as well as the destination (QSTA1 and QSTA2), between 7 (short period of time) and 20 seconds (long period). This interval was chosen based on the duration of well-known real video sequences.

There is a difficulty in obtaining code of other proposals, so the methodology adopted for the experiments considered two configurations for the simulation model: Regular (modules of IEEE $802.11 \mathrm{e} / \mathrm{s}$ ) and static configurations for classification and over-reserves for each AC; and MIRA-WMN, with MIRAWMN attached to the QMPs interacting with IEEE 802.11e/s for network resource provisioning and enforcement.

The methodology adopted to analyze the results was based on the data and control plans. Concerning former, traditional measures (i.e., mean throughput, flow delay and jitter) were traced to notice the quality behavior perceived by the packets of the flows composing the sessions. The mean throughput was analyzed to show to what extent the network is underused in a setting with no dynamic QoS enforcement, and also its help in the visualization of losses as a result of the congestion. Others aspects of MIRA-WMN performance were not considered is this work, since it would require other evaluation models (as prototyping). The experiments were repeated ten times, and the average of the outcome showed $95 \%$ confidence interval (not plotted in the graphs for better viewing of the results). Fig. 3 shows the mean throughput in the simulation model with MIRA-WMN and Regular configurations.

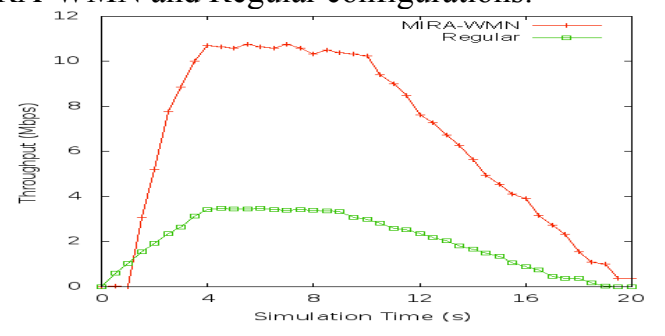

Figure 3. Mean throughput with Regular configuration

Results of Fig. 3 shows that MIRA-WMN allows fully allocating the bandwidth of the network, keeping QoS-aware sessions even under congestion settings. The tests with Regular configuration show lower flow propagation given the heavy packet losses due lack of control of exceeding flows injected in the WMN (as observed by packet loss results of Table I.)

TABLE I. LOSSES WITH MIRA-WMN AND REGULAR CONFIGURATION
\begin{tabular}{|c|c|c|}
\hline \multirow{2}{*}{ IEEE 802.11e AC } & \multicolumn{2}{|c|}{ Loss (\%) } \\
\cline { 2 - 3 } & Regular & MIR $\boldsymbol{A}-\boldsymbol{W} \boldsymbol{M} \boldsymbol{N}$ \\
\hline AC_BE & 50 & 32.9 \\
\hline AC_VI 1 & 30.8 & 21.7 \\
\hline AC_VI 2 & 49.9 & 33.6 \\
\hline AC_BI 3 & 50 & 38.9 \\
\hline
\end{tabular}

The numerical results (not shown in Fig. 3) notice that the simulation model with Regular configuration allowed AC_BEand $\mathrm{AC}$-VI-alike flows averaging $1.2 \mathrm{Mbps}$ and $0.7 \overline{\mathrm{M}} \mathrm{bps}$ respectively. This represents a use of only $30 \%$ of the network resources. On the opposite, the QoS provisioning and enforcement mechanisms of the MIRA-WMN enabled a use of network resources of $70 \%$ more than a Regular configuration setting. Moreover, the MIRA-WMN generated an amount of low signaling if compared to the traffic generated by the active 
sessions, 100kbps of signaling messages (corresponding to about $1 \%$ of the network resources). Taking into account the MIRA-WMN impact in sessions sensitive to QoS/QoE, the $100 \mathrm{~Kb}$ signaling traffic generated by the MIRA-WMN becomes relatively small.

The network delay results in the simulation model with Regular and MIRA-WMN configuration are depicted in Fig. 4. The delay measure is widely used to notice the quality perceived by packets along data paths. The QoE behavior is directly influenced by the QoS control in the network nodes, and the impact is perceived at the end users.

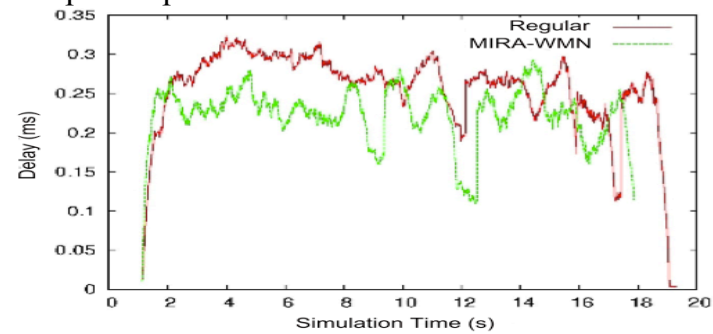

Figure 4. Delay results in the simulation experiments

The results of Fig. 4 show average delay of $0.22 \mathrm{~ms}$ (MIRA-WMN) and $0.25 \mathrm{~ms}$ (Regular). Moreover, the numerical results notices that the jitter results are of $0.003 \mathrm{~ms}$ (MIRA-WMN) and of $0.004 \mathrm{~ms}$ (Regular). The environment configured with MIRA-WMN reduces about $11.7 \%$ the delay, as well as $15.7 \%$ the jitter, compared to a Regular system. Even in situations of delay peak, the MIRA-WMN setting had a reduction of $12.5 \%$ o compared to the Regular configuration, considering that the delay peak with QoS was of $0.25 \mathrm{~ms}$ and the peak without the MIRA-WMN was of $0.32 \mathrm{~ms}$.

Although Table I shows a high percentage of losses with MIRA-WMN, this is due by the hostile feature of the wireless setting (limitation of radio transmission and high error rate, for example). Yet, the losses without the use of MIRA-WMN were caused by an uncontrolled situation experienced by network congestion. Therefore, subjective visual evaluations were carried out to show the MIRA-WMN impact on user's perception. Table II shows two frames randomly retrieved from videos transmitted in the simulations.

TABLE II. FRAME OF VIDEO NEWS WITH AND W/O MIRA-WMN

\begin{tabular}{|c|c|c|}
\hline CONFIGURATION & FRAME \# [12] & FRAME \# [17] \\
\hline REGULAR & & \\
\hline MIRA-WMN & & \\
\hline
\end{tabular}

Observe that the quality of the video transmitted in the simulations with Regular configuration was hindered by the losses experienced by the flow. There is a fake contradiction found in Table II in the analysis of losses of AC_VI1-alike traffic. This AC had fewer losses in a setting with Regular than MIRA-WMN configuration. The AC shows a better behavior as it occupied the network first than the other ACs, resulting in a smaller loss than others. Moreover, as previously explained, this AC underwent losses of packages with no retransmission, which certainly affected the content received by the user. It is important to conclude that when MIRA-WMN is used, the user received the multimedia session with excellent quality, while the Regular configuration tests showed poor quality.

\section{CONCLUSION AND FUTURE WORKS}

This work presents the Multi-Service Resource Allocation in Wireless Mesh Networks (MIRA-WMN) for efficient provisioning of resources in networks compatible with IEEE $802.11 \mathrm{e} / \mathrm{s}$ standard. The MIRA-WMN manages per-AC bandwidth provisioning and multicast channels in an integrated manner, and uses a low-cost signaling protocol. Moreover, the network is able to handle multi-user applications with ensured QoS/QoE without affecting its performance. For more reliability to the environment, the MIRA-WMN implements a low-cost resilience mechanism, providing intermittent sessions at an excellent quality level perceived by users. The MIRAWMN evaluation showed efficiency of mechanisms as it meets QoS requirements of sessions during its lifetime, combined to a low increase of traffic in the network with the signaling messages. More precisely, the MIRA-WMN showed $70 \%$ more efficiency in using bandwidth, $11.7 \%$ compared to the delay $(12.5 \%$ in peak) and $15.7 \%$ compared to Jitter, using $1 \%$ of resources available in the network. The graphic analysis showed that the MIRA-WMN provided users multimedia sessions with excellent quality perceived, while experiments with Regular configuration did not showed such. The MIRAWMN results pointed out as future work its analysis through prototyping for a more precise multi-user and performance study. Thus, the intention is to implement the MIRA-WMN in an actual setting that is already under implementation process.

\section{ACKNOWLEDGMENT}

The National Council for Scientific and Technological Development (CNPq) supported this work.

\section{REFERENCES}

[1] Hiertz, G. R et al, "IEEE 802.11s: The wlan mesh standard," in EEE Wireless Communications, pg. 104-111, 2010

[2] IEEE Computer Society, Standard for Information technology (2000). "Medium Access Control (MAC) Quality of Service Enhancements".

[3] P. Bristow, "Unicast vs. multicast over wireless: a cross-disciplinary mindshare for educational application researchers, " in 11th Annual Sigcse Conference on Innovation and Technology in Computer Science Education, New Yirk, NY, USA, 2006.

[4] X. Cheng, P. Mohapatra, S. Lee, S. Banerje, "MARIA: Interferenceaware admission control and qos routing in wireless mesh networks, " in IEEE Int. Conference on Coomunications, Beijing, China, 2008.

[5] G. Chauhan, S. Nandi, "QoS Aware Stable path Routing (QASR) Protocol for MANETs, " in $1^{\text {st }}$ International Conference on Emerging Trends in Engineering and Technology, Nagpur, India, 2008.

[6] J. Xue, P. Stuedi, G. Alonso, "Asap: An adaptive qos protocol for mobile ad hoc networks, " in IEEE Internation Symposium on Personal, Indoor and Mobile Radio Communications, Beijing, China, 2007.

[7] E. Cerqueira et al, "WiRA: An Approach to Resource Control in WiMAX Systems," in Next Generation Internet Networks, Krakow, Poland, 2008.

[8] A. Neto et al, "A Resource Reservation Protocol Supporting QoS-aware Multicast Trees for Next Generation Networks" in IEEE Symposium on Computers and Communications (ISCC), Aveiro, Portugal, 2007.

[9] E. Cerqueira et al, "QoS Support for Multi-user Sessions in IP-based Next Generation Networks," Springer/ACM Mobile Networks and Applications, Vol. 13, N 3-4, pp. 366-384, 2008 